\title{
A Cross-Layer Approach to Transmit Antenna Selection
}

\author{
José López Vicario, Student Member, IEEE, Miguel Ángel Lagunas, Fellow, IEEE, \\ and Carles Antón-Haro, Senior Member, IEEE
}

\begin{abstract}
In this paper, we investigate a cross-layer approach to transmit antenna selection capable of adapting the number of active antennas to varying channel conditions. We address a cross-layer methodology in the sense that the criterion for the selection of antenna subsets is the maximization of link layer throughput which takes into account characteristics both at the physical and link layers. In order to enhance system performance, adaptive modulation is included to jointly perform antenna selection and rate adaptation. Performance assessment is conducted in terms of link layer throughput and transmission delay.
\end{abstract}

Index Terms-Antenna selection, adaptive modulation, spatial multiplexing, vertical BLAST (V-BLAST) architecture, crosslayer optimization, Hybrid Automatic Repeat-reQuest (H-ARQ) protocols, multiple-input multiple-output (MIMO) systems.

\section{INTRODUCTION}

$\mathbf{N}$ EW wireless communications systems are aimed at satisfying the ever-increasing demand for high data rate communication services. In order to ensure high peak data rates, low latency and increased link throughput, one should often resort to advanced techniques. In the HighSpeed Downlink Packet Access (HSDPA) technical specification of UTRA [1], for instance, sophisticated transmission schemes such as Adaptive Modulation and Coding (AMC), fast Hybrid Automatic Repeat-reQuest (H-ARQ) and MultipleInput Multiple-Output (MIMO) are adopted. In particular, a spectrally-efficient spatial-multiplexing MIMO configuration [2] in combination with detection methods capable of removing spatial interference effects is used. Since, in general, computational complexity for Maximum Likelihood (ML) detection is not affordable, a V-BLAST (Vertical BLAST) architecture [3] is suggested as a viable alternative.

Contributions: In this paper, we investigate several crosslayer (CL) approaches to transmit antenna selection (AS). We address a CL methodology in the sense that the criterion for antenna subset selection is the maximization of link throughput which, clearly, takes into account characteristics both at the physical (PHY) and link layers, such as receiver structure,

Manuscript received May 19, 2004; revised April 26, 2005; accepted December 12, 2005. The associate editor coordinating the review of this letter and approving it for publication was D. Gesbert. This work was partially supported by the European Commission under projects IST-2002-507525 NEWCOM, IST-2002-508009 ACE; and the Catalan Government (DURSI) 2005FI 00003, SGR2005-00690.

J. L. Vicario and C. Antón-Haro are with the Centre Tecnològic de Telecomunicacions de Catalunya (CTTC), 08860 Castelldefells, Spain (e-mail: \{jose.vicario, carles.anton\}@cttc.es).

M. Á. Lagunas is with the Centre Tecnològic de Telecomunicacions de Catalunya (CTTC), 08860 Castelldefells, Spain, and also with the Department of Signal Theory and Communications, Technical University of Catalonia (UPC), 08034 Barcelona, Spain (e-mail: m.a.lagunas@cttc.es).

Digital Object Identifier 10.1109/TWC.2006.04339. packet size, modulation scheme and ARQ strategy. Some pioneering examples of cross-layer antenna selection can be found in [4]. Our contribution extends those initial works in order to encompass H-ARQ and AMC mechanisms. In other words, we will obtain a version of the scheme tailored to $\mathrm{H}$ ARQ needs that, in addition, is capable of jointly performing both antenna selection and adaptive modulation. Throughout the paper, the resulting CL approaches will be compared in terms of link layer throughput and transmission delay with other conventional schemes.

Relation to prior work: In a MIMO context, channel capacity bounds can be approached by conducting some sort of pre-processing on the transmit side. In those cases where reciprocity between the forward and reverse links does not hold, a feedback signalling channel is required to convey Channel State Information (CSI). Information sent over feedback channels, though, is subject to quantization effects, command delay and channel errors. In that context, transmit AS approaches [5] [6], emerged as an effective alternative featuring superior robustness in practical communication systems. As for the selection criteria, it is common practice to select the subset of transmit antennas maximizing channel capacity [7]. However, such capacity-based approaches, do not exploit all the information available in specific system scenarios concerning the actual schemes and algorithms in use at the physical and link layers. Therefore, maximizing channel capacity does not necessarily lead to an improved performance in terms of, for instance, data rates. For that reason, we adopt a throughput-based approach to AS (i.e., cross-layer design) that, as shown later, brings remarkable benefits in comparison with conventional capacity-based criteria.

Besides, AMC techniques can further improve spectral efficiency by adjusting transmission parameters to time-varying channel conditions [8], in particular when applied to MIMO configurations [9]. Adaptive modulation techniques in combination with AS strategies were introduced in e.g., [10]; by switching off antennas elements unable to meet specific QoS requirements the overall interference level can be reduced and, thus, higher constellation sizes can be supported by the remaining ones. When adopting a throughput-based criterion, though, the integration of an adaptive modulation scheme with the AS strategy comes in a very natural way. As a consequence, unnecessary separate optimizations (often resorting to heuristic criteria) are avoided.

Organization: The corresponding system model is presented in Section II. In Section III, the throughput-based criterion is introduced, and is further enhanced to incorporate adaptive modulation in Section IV. Finally, some simulation results and conclusions are provided in Sections V and VI, 


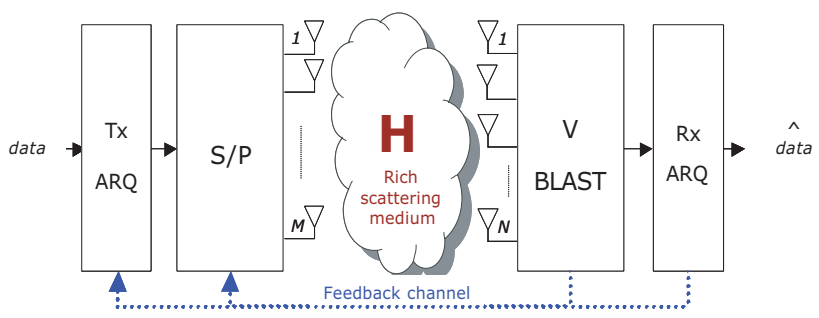

Fig. 1. Block diagram of the communication system.

respectively.

\section{System Description}

Consider the downlink of a cellular wireless system where the signals corresponding to different users are separated by using spreading codes. For a transmission link between an $M$ antenna Base Station and an $N$-antenna terminal (see Fig. 1) where an ideal spreading/despreading process is assumed, the received signal can be modelled as:

$$
\mathbf{r}=\mathbf{H}_{m}\left(\mathbf{m}_{m}\right) \mathbf{a}+\mathbf{n}
$$

where $\mathbf{H}_{m}\left(\mathbf{m}_{m}\right)$ is the $N \times m$ matrix defined by the $m$ columns of $\mathbf{H}$ corresponding to the subset of active transmit antennas given by vector $\mathbf{m}_{m}\left(m_{i} \in\{0,1\} ; i=1 . . M\right)$, a denotes the transmitted symbols vector drawn out of the selected constellation, and $\mathbf{n}$ stands for an additive Gaussian noise vector of complex, random variables with zero mean and variance $\sigma^{2}$ (accounting for both intra- and inter-cell interference, i.e., in the presence of long scrambling codes). For the ease of notation, we will denote $\mathbf{H}_{m}\left(\mathbf{m}_{m}\right)$ as $\mathbf{H}_{m}$, that is, we will not explicitly state the dependence of the channel matrix on vector $\mathbf{m}$. Channel response is assumed to exhibit block Rayleigh flat-fading characteristics. Besides, it is also assumed that perfect Channel State Information (CSI) is available at the receive side, where a V-BLAST detection scheme is used [3]. At the transmit side, power is evenly distributed among active antennas, that is, it is proportional to $1 / m$.

In close alignment with HSDPA specifications, we consider a Type III H-ARQ scheme at the link layer. More precisely, we adopt an N Stop-and-Wait (NSAW) re-transmission protocol (which minimizes signalling and buffering requirements at the UE) along with a chase combining strategy [1] for retransmitted packets. Packet combining is done by averaging soft symbols at the output of the V-BLAST detector. Therefore, the resulting symbol estimates after $p-1$ consecutive retransmissions can be expressed as $\mathbf{y}_{p}=\frac{1}{p} \sum_{i=1}^{p} \mathbf{y}_{v b, i}$, where $\mathbf{y}_{v b, i}$ denotes the soft-symbol vector at the output of the VBLAST scheme at the $i$-th transmission.

\section{Cross-layer Transmit Antenna Selection}

A widely-used [7] antenna selection criterion for spatial multiplexing consists in selecting the transmit antenna subset maximizing channel capacity:

$$
C_{m}=\log _{2} \operatorname{det}\left[\mathbb{I}_{N}+\frac{\rho}{m} \mathbf{H}_{m} \mathbf{H}_{m}^{H}\right]
$$

where $\mathbb{I}_{N}$ is the $N \times N$ identity matrix and $\rho$ is the average signal-to-noise ratio per receive antenna. With some abuse of notation, we will refer to such approaches as PHY-layer only, in the sense that only PHY layer parameters (i.e., channel response and SNR) are explicitly taken into account.

In order to maximize link throughput and, ultimately, enhance overall performance, additional information such as receiver structure, packet size, modulation scheme or HybridARQ strategy, etc., could be exploited as well, thus leading to a cross-layer approach. As a previous step, an expression for the link throughput will be obtained in subsequent paragraphs.

For symbols conveyed in the first packet transmission (recall the H-ARQ strategy) which are detected in the $k^{t h}$ iteration (layer) of the V-BLAST algorithm, we can define the post-filtering symbol energy-to-noise plus interference spectral density ratio $\left(E_{s} / N_{o}\right)$ as:

$$
\rho_{k, 1}=\frac{E\left[\left|a_{k}\right|^{2}\right]}{\sigma^{2}\left\|\mathbf{w}_{k, 1}\right\|^{2}} \quad k=1,2, \ldots, M,
$$

being $a_{k}$ the transmitted symbol, and $\mathbf{w}_{k, 1}$ the V-BLAST zeroforcing spatial filter for the $k^{\text {th }}$ layer. When considering a chase-combining strategy the effective $E_{s} / N_{o}$ after $p$ transmissions (i.e., $p-1$ re-transmissions) can be expressed as $\rho_{k, p}=\alpha^{p-1} p \rho_{k, 1}$, where $\alpha$ is the chase-combining efficiency factor, that models the combining gain loss with respect to the theoretical model [11]. Notice that, expression $\alpha^{p-1} p$ is concave on the number of packet transmissions, $p$ (when relaxing $p$ to be a continuous variable). Therefore, there is no point in increasing the number of retransmissions when the maximum is achieved ( $p \leq P$, being $P$ the optimum number of packet transmissions).

By disregarding error propagation in previous layers and taking into account the specific modulation scheme in use, $R$, a closed-form expression for the Symbol Error Rate (SER) at each V-BLAST layer can be obtained, $S E R_{k, p}=\gamma\left(\rho_{k, p}, R\right)$. From that, we can derive an expression for the uncoded Packet Error Rate (PER) ${ }^{1}$. Since as many $L$-symbol packets as actual antennas are to be transmitted in parallel, the resulting PER expression for the $L \cdot m$-symbol packet being transmitted from the subset of antennas defined by vector $\mathbf{m}$, is given by:

$$
\operatorname{PER}_{p}(\mathbf{m})=1-\left[\prod_{k=1}^{m}\left(1-S E R_{k, p}\right)\right]^{L}
$$

where we have assumed independent errors to occur.

The link layer throughput is measured as the effective number of correctly received bits at the link layer per channel use. Therefore, packet retransmissions due to the H-ARQ strategy must be taken into consideration. For the SAW protocol, the throughput expression can be written as [14]:

$$
\eta_{S A W}=\frac{l \cdot b}{W \cdot \mathrm{E}[p]}
$$

\footnotetext{
${ }^{1}$ For a coded system, a different PER expression would result. In the case of convolutional codes, for instance, one could resort to the accurate and simple approximations in [12]. Since, this work is aimed at illustrating a method to encompass both PHY and LINK level parameters in the cost function and for the sake of simplicity, we will restrict ourselves to uncoded systems.
} 
TABLE I

COMPUTATIONAL COMPLEXITY ANALYSIS FOR A 3X3 MIMO SYSTEM. THE FIRST COLUMN REFLECTS THE OPERATION COUNT FOR THE ANTENNA SELECTION PART (IF APPLICABLE) AND THE COMPUTATION OF SPATIAL FILTERS. THE SECOND COLUMN ACCOUNTS FOR THE SPATIAL FILTERING OF $L=160$-SYMBOL VECTORS PACKETS. THE FOURTH COLUMN STANDS FOR THE NUMBER OF OPERATIONS PER SECOND CONSIDERING PACKETS OF 0.667 MS TIME DURATION.

\begin{tabular}{|c|c|c|c|c|}
\hline & $\begin{array}{c}\text { Antenna selection + computation } \\
\text { of spatial filters [FLOPS] }\end{array}$ & $\begin{array}{c}\text { Spatial filtering } \\
\text { [FLOPS] }\end{array}$ & $\begin{array}{c}\text { Total } \\
\text { [FLOPS] }\end{array}$ & $\begin{array}{c}\text { Complexity } \\
\text { [MFLOPS/s] }\end{array}$ \\
\hline Top-down [13], without AS & 344 & 18,240 & 18,584 & 27.9 \\
\hline Top-down, with AS & 626 & 18,240 & 18,866 & 28.3 \\
\hline
\end{tabular}

where $W$ stands for the round-trip delay expressed in number of slots, $\mathrm{E}[p]$ is the average number of packet retransmissions, $l$ is the ratio of information symbols per packet and $b$ the number of bits per symbol, according to the modulation scheme in use. Then, by obtaining an estimate of the mean number of packet retransmissions, considering that $m$ streams are spatially multiplexed and the fact that $N_{S A W}$ processes are run in parallel (in order to minimize idle time), an expression for the link layer throughput can be found:

$$
\begin{aligned}
& \eta(\mathbf{m})=\frac{N_{S A W} \cdot l \cdot m \cdot b}{W \cdot \mathrm{E}[p]} \\
& \frac{l \cdot m \cdot b \cdot N_{S A W} / W}{\left(1-P E R_{1}(\mathbf{m})\right)+\sum_{p=2}^{P}\left[p\left(1-P E R_{p}(\mathbf{m})\right) \prod_{t=1}^{p-1} P E R_{t}(\mathbf{m})\right]}
\end{aligned}
$$

The above expression is used by the receiver in order to determine the optimal subset of transmit antennas, that is, the one maximizing link throughput. After that, the $M$ dimensional vector with the active antenna subsets is signalled to the transmitter over the feedback channel.

Given the highly non-linear nature of both the throughput expression and the maximization problem itself, a straightforward approach consists in checking every single combination of transmit antennas. In that context, main contributions to complexity result from the computation of the vector norms of the linear receivers (i.e., $\left\|\mathbf{w}_{k, 1}\right\|^{2}$ ) which are needed in the throughput scoring process. In the case of V-BLAST schemes, a series of inverse matrices are needed to obtain those vector norms. However, such set of inverse matrices can be efficiently computed by invoking the block matrix inversion lemma and, by doing so, a lower-complexity recursive approach, named the top-down algorithm, was derived by the authors in [13]. For a moderate number of transmit and receive antennas, the additional cost of introducing transmit AS mechanisms (with respect to a scheme with a fixed number of transmit antennas) turns out to be only $1.52 \%$ (see Table I). Notice that, in contrast with the recursive sub-optimal approaches derived in [6] and [7], the top-down approach is capable of identifying the optimal antenna subset (in a CL sense) since it incurs no inaccuracy in the recursive computation of vector norms or in the exploration of different antenna subsets.

Finally, in order to adapt the proposed AS methodology to the H-ARQ protocol in use, the following restrictions apply:

- The actual subset of transmit antennas has to remain unchanged during packet retransmissions. This is needed in order to appropriately add-up packets with the chasecombining approach. This limitation, though, has little impact on performance in a context of slow moving terminals where the same antenna subset is valid over several consecutive frames.

- Packets being transmitted in parallel from different antennas must be accepted/discarded as a block. Since no numbering strategy is adopted by the SAW protocols, it is not possible to retransmit part of the $m$-packet block. As shown in [15], block discarding caused by, possibly, a single corrupted packet, has little impact on system performance provided that a throughput maximization strategy is adopted.

- Since multiple SAW processes are run in parallel, the same number of antenna selection processes can be conducted independently.

\section{InClusion of AdAptive Modulation Schemes}

In this section, we further enhance the antenna selection algorithms presented in Section III with the inclusion of adaptive modulation (AM) schemes. As will be shown later, this can be done in a more natural and integrated way for the cross-layer approach.

More precisely, it consists in jointly determining (by exhaustive search) both the best subset of transmit antennas along with the corresponding modulation scheme which maximize the link throughput given by Eq. 6. In order not to further increase complexity and keep signalling in the feedback channel low, a common modulation scheme will be shared by every single active antenna. In a CL antenna selection context, though, this additional constraint does not have a major impact in performance as discussed later in Section V. It is worth noting that the (linear) increase in computational burden resulting from throughput scoring over different modulation schemes can be considered a minor issue in comparison with the contributions from the computation of vector norms.

As an extension of the PHY-layer antenna selection methodology presented in section III, an algorithm incorporating adaptive modulation procedures is proposed next. First, the transmit antennas sub-set is selected according to the mentioned PHY-layer strategy. After that, the associated modulation scheme has to be determined on the basis of PHY layer parameters only. In order to minimize the number of packets dropped for the resulting antenna configuration, we adopt a PER-based criterion. In particular, the largest constellation size for which the resulting PER is below a specific threshold, $P E R_{t h}$ will be selected. Again, the same modulation scheme will be shared by all active antennas. 


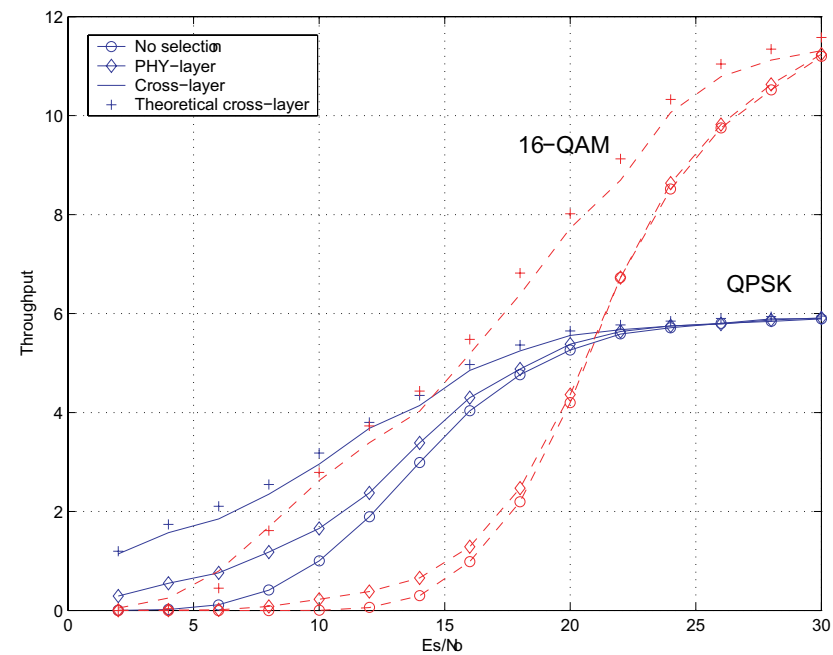

Fig. 2. Throughput vs. $E_{s} / N_{o}$ for the throughput-based (cross-layer) and capacity-based (PHY-layer) antenna selection mechanism in a $3 \times 3$ MIMO configuration $\left(W=3, N_{S A W}=3\right)$.

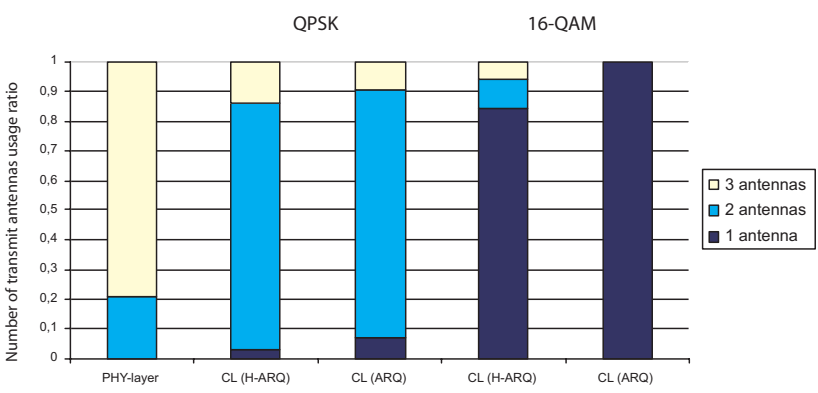

Fig. 3. Relative frequency of 1,2 and 3-antenna combinations for different antenna selection strategies and modulation schemes. No chase combining of retransmitted packets was conducted for bars labelled with 'ARQ' $\left(E_{s} / N_{o}=12 \mathrm{~dB}\right)$.

\section{COMPUTER Simulation RESUlTS}

As far as computer simulations are concerned, we will consider a scenario of low mobility terminals $\left(v_{U E}=3\right.$ $\mathrm{km} / \mathrm{h}$ ) where $2 \mathrm{~ms}$ frames are divided into three slots, each featuring a $L=160$-symbol packet (adopted from HSDPA). We will assume a round-trip delay of $W=3$ slots and, accordingly, we will run $N_{S A W}=W=3$ concurrent SAW processes. The FeedBack channel Information (FBI) field of HSDPA will be used to convey AS commands to the transmit side over an error- and delay-free feedback channel. Since only one feedback bit per uplink control slot is available, AS commands are limited to three bits per uplink frame. As for the maximum number of transmit and receive antennas are set to $M=3$ and $N=3$, respectively. Concerning the chase combining reliability parameter, $\alpha$, it was empirically determined from a scenario without AS (by means of extensive Montecarlo simulations and least-squares fitting) and found to be equal to 0.74 and 0.72 for QPSK and 16-QAM respectively, thus setting the maximum number of packet transmissions to $P=4$.

In Figure 2, performance in terms of link layer throughput vs. $E_{s} / N_{o}$ for the AS methodologies based on capacity (PHY) and throughput (CL) measures is compared. For benchmarking, an additional curve for a system without performing

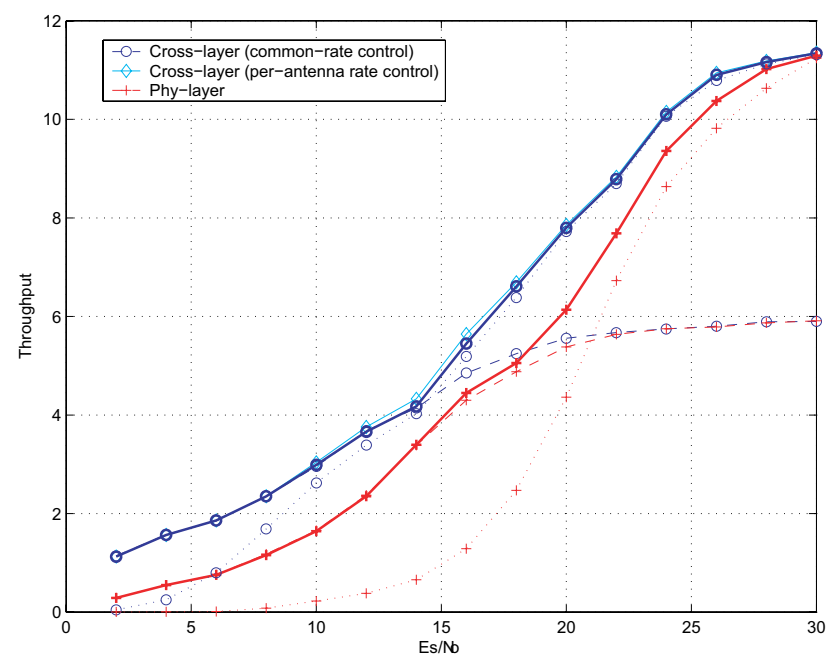

Fig. 4. Performance in terms of throughput vs. $E_{s} / N_{o}$ for the throughputbased (cross-layer) and capacity-based (PHY-layer) antenna selection schemes with adaptive modulation. Solid lines: adaptive modulation (with commonand per-antenna rate control, dashed lines: QPSK, dotted lines: 16-QAM).

antenna selection is included, as well. In order to validate the expression proposed in section III for the link layer throughput, we also include results obtained by direct evaluation of Eq. 6 (curves labelled with theoretical). It becomes apparent that the throughput-based criterion significantly outperforms its capacity-based counterpart for the whole range of $E_{s} / N_{o}$ and for both modulation schemes. The larger the constellation size (16-QAM) the wider the gap between curves for solutions maximizing capacity and throughput. Clearly, the former one does not explicitly consider the actual modulation scheme whereas the latter takes that into account (along with packet size and other transmission parameters). As a result, the throughput-based criterion is capable of further concentrating transmit power on the best channels when using 16-QAM. By doing so, the transmit power is efficiently exploited and the detection procedure can be improved because error propagation between layers is reduced. Indeed, little or no improvement is achieved by the capacity-based solution with respect to the no antenna selection case. Such behavior can also be appreciated in Fig. 3 where a histogram of antenna usage ratio is shown for both optimization criteria. Clearly, the capacity-based method tends to use a larger number of antennas whereas throughput criterion is more conservative, in particular when no packet combining method is used.

Figure 4 shows how both AM extensions of the PHY- and cross-layer antenna selection algorithms make the most of the different modulation schemes (QPSK and 16 QAM). In the PHY-layer approach, the threshold packet error rate was empirically set to $P E R_{t h}=0.2$ in order to maximize the resulting link throughput. To start with, one can observe that the resulting AM-PHY throughput curve runs well below its AM-CL counterpart for the whole range of $E_{s} / N_{o}$ ratios. Actually, there is a 3-4 $d B$ shift in average $E_{s} / N_{o}$ (with both schemes operating in a common-rate configuration). As for the gain resulting from the inclusion of per-antenna rate control, it turns out to be rather limited. In the low and high$E_{s} / N_{o}$ regions, selecting a common modulation scheme for all 


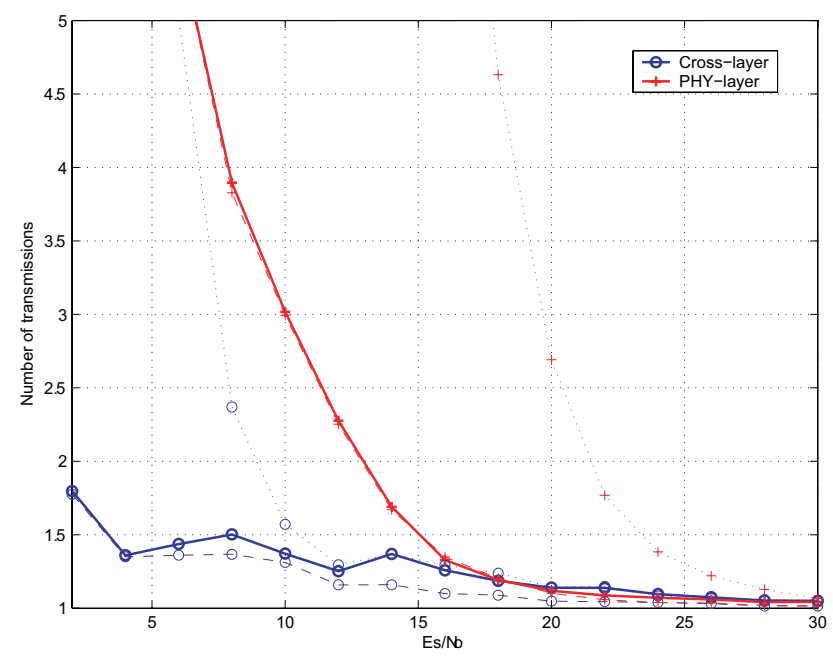

Fig. 5. Average number of retransmissions with and w/out adaptive modulation schemes for both antenna selection criteria. Solid lines: with adaptive modulation, dashed lines: QPSK, dotted lines: 16-QAM.

active antennas (the lowest and the highest, respectively) does definitely make sense. In the mid- $E_{s} / N_{o}$ region, additional degrees of freedom are provided by the antenna selection mechanisms, thus resulting in an improved granularity in terms of effective data rates. In other words, the fact that both the constellation size and the actual transmit antennas are jointly selected, partially compensates for the losses resulting from failing to adapt constellation size on a per-antenna basis. This is not possible in a system where the number of transmit antennas is kept constant and, hence, the gap becomes larger [16].

In terms of transmission delay (Fig. 5), the AM-CL approach, in combination with H-ARQ mechanisms, provides a means to effectively keep the number of individual packet transmissions low for the whole range of signal to noise ratios (less than 2). Conversely, the number of packet retransmissions grows exponentially in the low $-E_{s} / N_{o}$ region for the AMPHY approach. This fact indicates that error propagation cannot be circumvented by including an AM scheme since the initial selection of transmit antennas (on the basis of capacity criterion) is not appropriate.

\section{CONCLUSIONS}

In this paper, a cross-layer approach to transmit antenna selection on the basis of a throughput-based criterion was presented. For reference purposes, a conventional capacity-based antenna selection scheme was considered as well. Enhanced versions aimed at jointly performing antenna selection and rate adaptation were derived for both the CL and PHY-layer approaches in order to exploit better the available modulation schemes. In terms of throughput, the cross-layer approach outperformed the PHY-layer one for the whole range of signalto-noise ratios with gains of up 3-4 dB in average $E_{s} / N_{o}$.
Substantial benefits resulted in terms of transmission delay too.

Future work in this field will encompass an extension to multi-user scenarios with the derivation of cross-layer scheduling techniques in combination with antenna selection methods.

\section{ACKNOWLEDGMENT}

The authors wish to thank Xavier Mestre for reading the paper and for his helpful comments. They would also like to thank the anonymous reviewers for their comments on the paper.

\section{REFERENCES}

[1] "Physical layer aspects of UTRA high speed downlink packet access," Technical Specification, 3rd Generation Partnership Project, vol. 25.848, March 2001, release 4.

[2] G. Foschini and M. Gans, "On limits of wireless communications in a fading environtment when using multiple antennas," Wireless Pers. Commun., vol. 6, no. 3, pp. 311-335, Mar. 1998.

[3] G. Golden, C. Foschini, R. Valenzuela, and P. Wolniansky, "Detection algorithm and initial laboratory results using v-BLAST space-time communication architecture," Electron. Lett., vol. 35, no. 1, pp. 14-16, Jan. 1999.

[4] A. Milani, V. Tralli, and M. Zorzi, "Improving protocol performance in BLAST-based wireless systems using channel adaptive antenna selection," in IEEE Proc. Vehicular Technology Conference Spring, vol. 1, pp. 409-413, 2002.

[5] A. F. Molisch, "MIMO systems with antenna selection - an overview," in Proc. Radio and Wireless Conference, Boston (USA), pp. 167-170, Aug. 2003.

[6] M. Gharavi-Alkhansari and A.B.Gershman, "Fast antenna subset selection in MIMO systems," IEEE Trans. on Signal Processing, vol. 52, no. 2, pp. 339-347, Feb. 2004

[7] A. Gorokhov, "Antenna selection algorithms for MEA transmission systems," in Proc. IEEE Int. Conf. Acoust., Speech, Signal Processing, vol. 3, pp. 2857-2860, May 2002.

[8] A. Goldsmith and S. Chua, "Variable-rate variable-power MQAM for fading channels," IEEE Trans. on Commun., vol. 45, pp. 1218-1230, Oct. 1997.

[9] S. Cautreaux, P. Driessen, and L. Greensteins, "Data throughputs using multiple-input multiple-output (MIMO) techniques in a noise-limited cellular environment," IEEE Trans. on Wireless Commun., vol. 1, no. 2, pp. 226-234, April 2002.

[10] H. Zhuang, L. Dai, S. Zhou, and Y. Yao, "Low complexity per-antenna rate and power control approach for closed-loop V-BLAST," IEEE Trans. on Wireless Commun., vol. 51, no. 11, pp. 1783-1787, Nov. 2003.

[11] F. Frederiksen and T. E. Kolding, "Performance and modeling of WCDMA/HSDPA transmission/H-ARQ schemes," in Proc. IEEE Vehicular Technology Conference Fall, vol. 1, pp. 472-476, Sept. 2002.

[12] Q. Liu, S. Zhou, and G.B. Giannakis, "Cross-layer combining of adaptive modulation and coding with truncated ARQ over wireless links," IEEE Trans. on Wireless Communications, vol. 3, no. 5, pp. 1746-1755, Sept. 2004.

[13] J. López-Vicario, C. Mecklenbräuker, and C. Antón-Haro, "Reducedcomplexity methods for throughput maximization in MIMO channels," in Proc. IEEE International Conference on Communications, vol. 5, pp. 2678-2682, June 2004.

[14] S. Lin, D. Costello, and M. J. Miller, "Automatic-repeat-request errorcontrol schemes," IEEE Commun. Mag., vol. 22, no. 12, pp. 5-17, Dec. 1984.

[15] J. López-Vicario and C. Antón-Haro, "Throughput optimisation for MIMO systems via cross-layer designs," in Proc. 9th World Wireless Research Forum, July 2003.

[16] — - "Joint transmit antenna selection and adaptive modulation in cross-layer oriented designs for HSDPA systems," in Proc. IEEE Sensor Array and Multichannel Signal Processing Workshop, pp. 523-527, July 2004. 